\title{
Sevoflurane induces neurotoxicity in young mice through FAS/FASL signaling
}

\author{
Q. Song ${ }^{1 *}$, Y.L. Ma ${ }^{1 *}$, J.Q. Song ${ }^{1}$, Q. Chen ${ }^{1}$, G.S. Xia ${ }^{1}$, J.Y. Ma1, F. Feng ${ }^{1}$, \\ X.J. Fej $\mathrm{i}^{2,3}$ and Q.M. Wang ${ }^{1}$ \\ 'Department of Anesthesiology, Zhengzhou Central Hospital, Zhengzhou, \\ Henan Province, China \\ 2Department of Anesthesia and Critical Care, \\ Massachusetts General Hospital and Harvard Medical School, Charlestown, MA, USA \\ ${ }^{3}$ Department of Hospital Infections, \\ Shuguang Hospital Affiliated with Shanghai University of Traditional Chinese Medicine, \\ Shanghai, China \\ *These authors contributed equally to this study. \\ Corresponding author: X.J. Fei / Q.M. Wang \\ E-mail: weiymzhl@aliyun.com / min2178@163.com \\ Genet. Mol. Res. 14 (4): 18059-18068 (2015) \\ Received August 17, 2015 \\ Accepted October 15, 2015 \\ Published December 22, 2015 \\ DOI http://dx.doi.org/10.4238/2015.December.22.32
}

\begin{abstract}
Sevoflurane, the most widely used anesthetic in clinical practice, has been shown to induce apoptosis, inhibit neurogenesis, and cause learning and memory impairment in young mice. However, the underlying mechanism is still unknown. In this study, wild-type and the FAS- or FAS ligand (FASL)knockout mice (age 7 days) were exposed to sevoflurane or pure oxygen. Western blotting was used to examine the expression of FAS protein. Terminal deoxynucleotidyl transferase dUTP nick-end labeling (TUNEL) and bromodeoxyuridine (BrdU) staining were employed to quantify the apoptotic cells and newborn cells in the hippocampus and Morris water maze (MWM) in order to evaluate learning and memory status. Sevoflurane significantly increased the expression of FAS protein in wild-type mice. Compared to FASand FASL-knockout mice treated with sevoflurane, sevoflurane-treated wildtype mice exhibited more TUNEL-positive hippocampal cells and less BrdU-
\end{abstract}


positive hippocampal cells. The MWM showed that compared with FAS- and FASL-knockout mice treated with sevoflurane, sevoflurane treatment of wildtype mice significantly prolonged the escape latency and reduced platform crossing times. These data suggest that sevoflurane induces neurotoxicity in young mice through FAS-FASL signaling.

Key words: Sevoflurane; Apoptosis; Neurogenesis; Fas/FasL

\section{INTRODUCTION}

Sevoflurane is a commonly used clinical anesthetic because it is fast acting, produces less irritability, and stabilizes the hemodynamic status of the patient. However, it has been shown to induce cognitive impairment in rodents and might be associated with a higher incidence of learning and memory impairment in humans, leading to increased cost, morbidity, and mortality (Nikizad et al., 2007; Wilder et al., 2009; Zhang et al., 2013a). In recent studies, researchers have shown many adverse effects caused by sevoflurane on the developing brain especially in the embryonic period, neonatal period, and infancy (Shen et al., 2013; Zheng et al., 2013; Zhang et al., 2013b). They concluded that sevoflurane could increase neurodegeneration in the developing hippocampus of fetal rats. However, the detailed mechanisms underlying the deleterious effect of sevoflurane on brain development are still unclear.

Apoptosis is commonly induced in the cell via the FAS singling pathway (Medana et al., 2001). The FAS receptor protein is also known as APO-1 or CD95. It belongs to the subgroup of the tumor necrosis factor receptor (TNF-R) family that can trigger apoptosis. Its physiological ligand, FASL (CD95L), is a member of the corresponding TNF cytokine family (Huang et al., 2001). FAS and FASL play critical roles in immune system function, especially in the killing of pathogen-infected target cells and the death of obsolete, potentially deleterious, and autoreactive lymphocytes (Krammer, 2000). Further, the FAS signaling machinery is also involved in non-apoptotic processes, including cellular activation, differentiation, and proliferation (Strasser et al., 2009). Various drugs and substances can induce neuronal apoptosis through FASL-FAS signaling and may involve GSK-3 inhibition. For instance, the commonly used GSK-3 inhibitor, lithium, reportedly facilitates apoptotic signaling induced by the activation of the FAS death domaincontaining receptor (Gomez-Sintes and Lucas, 2013).

In a previous study, sevoflurane induced caspase-3 activation and apoptosis (Shen et al., 2013). FASL-FAS signaling is a major regulator of apoptosis, however, whether sevoflurane can induce apoptosis through the FASL-FAS signaling pathway is unknown. In this study, we found that exposure to sevoflurane induced cognitive impairment in young mice and activated FASL-FAS signaling. Young FAS and FASL-knockout mice exhibited attenuated sevoflurane-induced neuron apoptosis, neurogenesis inhibition, and learning and memory impairment. This finding may pave the way for the development of a therapeutic approach to prevent or treat sevoflurane-induced neurotoxicity.

\section{MATERIAL AND METHODS}

\section{Animals}

Six-day-old C57/BL wild-type mice were obtained from the specific pathogen-free animal center at Zhengzhou Central Hospital, which is affiliated with Zhengzhou University. All animal 
experiments were approved by the Animal Care Committee of Zhengzhou Central Hospital Affiliated with Zhengzhou University.

Mice were treated with 3\% sevoflurane from postnatal day 6 (P6) to P8, as described in our previous study, and a behavioral test was performed from P30 to P34. The anesthetic (sevoflurane) plus 60\% oxygen (balanced with nitrogen) was administered, as in the previous study (Shen et al., 2013).

\section{MWM test}

The MWM test was conducted in a circular tank (diameter: $1.8 \mathrm{~m}$ ) that was filled with opaque water. A platform $(11 \times 11 \mathrm{~cm})$ was submerged below the water's surface in the center of the target quadrant. The swimming path of each mouse was recorded using a video camera and analyzed using the Videomot 2 software. For each training session, mice were placed into the maze from four random points in the tank. They were allowed to search for the platform for $60 \mathrm{~s}$. If they did not find the platform within $60 \mathrm{~s}$, they were gently guided towards it. Mice were allowed to remain on the platform for $15 \mathrm{~s}$. Two training trials were performed every day, and the latency for each trial was recorded for analysis. During the memory test (probe test), the platform was removed from the tank, and the mice were allowed to swim in the maze for $60 \mathrm{~s}$.

\section{TUNEL staining for DNA fragmentation}

Mouse brains were removed after treatments with or without sevoflurane and stored at $4^{\circ} \mathrm{C}$ in $4 \%$ paraformaldehyde. Serial coronal sections $(10 \mu \mathrm{m})$ were cut on a cryostat $(\mathrm{CM} 3050$ $\mathrm{S}$, Leica Biosystems, Germany) and mounted on coverslips. Sections were permeabilized with proteinase $\mathrm{K}$ solution $(20 \mu \mathrm{g} / \mathrm{mL})$ for $20 \mathrm{~min}$. Terminal deoxynucleotidyl transferase (TdT) and dUTP (11684817910, Roche) were added to the sections and incubated in a humidified chamber at $37^{\circ} \mathrm{C}$ for $2 \mathrm{~h}$. The reaction was then stopped and the sections were stained with 4'6'-diamidino-2phenylindole (C1002, Beyotime, China) for $10 \mathrm{~min}$. Then, coverslips were mounted on glass slides with anti-fade mounting medium (P0126, Beyotime, China). Finally, the sections were analyzed under a light microscope (ECLIPSE TI-SR, NIKON, Japan) with $5 \mathrm{X}$ and 20X objective lenses, and photographs of the sections were taken. The number of TdT dUTP nick-end labeling (TUNEL)positive cells was counted using the Image $\mathrm{J}$ software by an investigator who was blinded to the experimental design.

\section{BrdU immunohistochemistry}

Mouse brains were removed after treatment with or without sevoflurane. For the detection of newborn cells in the hippocampal dentate gyrus, BrdU-specific immunohistochemistry was performed with the BrdU Immunohistochemistry Kit (ab125306). The brain sections were deparaffinized and incubated with the quenching solution for $10 \mathrm{~min}$. Two drops of trypsin enzyme were then added to each slide and incubated at room temperature about $25^{\circ} \mathrm{C}$ for $10 \mathrm{~min}$, followed by a 3-min rinse in distilled water. Two drops of the denaturing solution were added to each slide and incubated at room temperature for $30 \mathrm{~min}$. The sections were then incubated with blocking buffer at room temperature for $10 \mathrm{~min}$, BrdU antibody (ab6362; 1:200) at room temperature for 60 min, and streptavidin-horseradish peroxidase (HRP) conjugate at room temperature for $10 \mathrm{~min}$. The newborn cells were counted using the Image $\mathrm{J}$ software. 


\section{Western blotting}

Frozen mouse hippocampal tissues were homogenized, and the lysates were prepared in ice-cold lysis buffer. Total protein was collected and normalized for equal amounts, as measured by the bicinchoninic acid method. Seventy micrograms of protein from each sample was separated on a sodium dodecyl sulfate-polyacrylamide gel and transferred to polyvinylidene fluoride membranes. The membranes were blocked with $5 \%$ nonfat milk and incubated overnight with the primary antiFAS antibody (1:1000; ab82419, Abcam, Cambridge, MA, USA) and anti- $\beta$-actin antibody (1:5000; ab156302, Abcam) at $4^{\circ} \mathrm{C}$, followed by incubation with the suitable HRP-conjugated secondary antibody for $4 \mathrm{~h}$. Beta-actin protein was immunodetected as the internal standard.

\section{Statistical analysis}

Data regarding biochemical changes are reported as means \pm standard deviation (SD). The data regarding changes in escape latency were a better description of deviation from normality, and are reported as means \pm standard error of the mean (SE). Because the data for platform crossing times were not a good descriptor of deviation from normality, they are reported as median and interquartile range. Interactions of time and group factors in a two-way analysis of variance (ANOVA) with repeated measurements were used to analyze the difference in learning curves (based on escape latency) between mice in the control group and mice in the anesthesia group in the MWM. The Mann-Whitney U-test was used to determine differences in platform crossing times. The normality test shows that the data were normally disturbed. Finally, the Student two-sample $t$-test was used to determine differences in TUNEL-positive cells and BrdU-positive cells between control and anesthesia groups. Values of $\mathrm{P}<0.05$ were considered to be statistically significant. The GraphPad software Prism 5 (San Diego, CA, USA) was used to analyze the data.

\section{RESULTS}

\section{Multiple sevoflurane exposure induced cognitive impairment in young mice and activated FASL-FAS signaling}

Mice were treated daily with $3 \%$ sevoflurane for $2 \mathrm{~h}$ from P6 to P8, after which they were tested in the MWM from P30 to P34. A comparison of the time that each mouse took to reach the platform during reference training (escape latency) showed that there was a statistically significant interaction between time and group (Figure $1 \mathrm{~A})(P=0.0028$, two-way ANOVA with repeated measurement). The post hoc Bonferroni test showed that the mice that received sevoflurane anesthesia had longer escape latency than the mice following the control condition on P33 and P34. Furthermore, sevoflurane-treated mice $(N=15)$ had decreased platform crossing times (median, 3; interquartile range, 2.5-5.5), which represented the number of times that each mouse had crossed the location of the absent platform at the end of reference training, as compared to control mice $(\mathrm{N}=15)$ (median, 6; interquartile range 4-8) (Figure 1B) $(P=0.042$, Mann-Whitney U-test). There was no significant difference in mouse swimming speed between the mice in the sevoflurane anesthesia group and the mice in the control group (data not shown). These data suggested that multiple sevoflurane exposures in young mice might induce cognitive impairment after 3 weeks. Sevoflurane also upregulated the expression of FAS as compared to the control condition in hippocampal tissues (Figure 1C). 
A



B

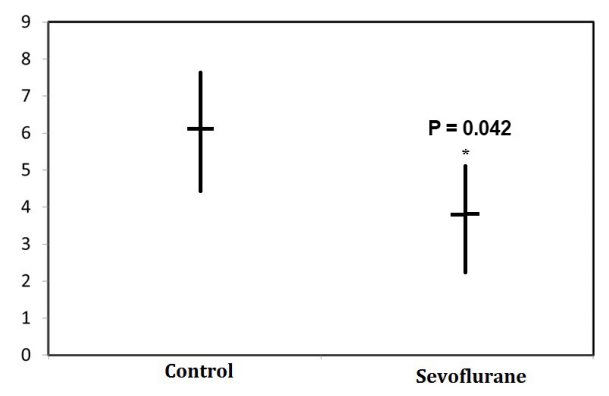

C

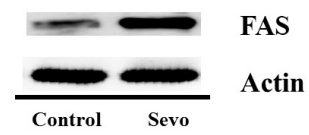

Figure 1. Exposure to $3 \%$ sevoflurane daily for 3 days induces cognitive impairment in six-years-old mice. A. Mice that were exposed to $3 \%$ sevoflurane for $2 \mathrm{~h}$ daily from postnatal day 6 (P6) to P8 exhibited increased escape latency at P30-P34 in the Morris water maze (MWM), compared with the control mice (control, $N=15$; sevoflurane, $N=15$ ). Results from two-way analysis of variance (ANOVA) with repeated measurement analysis show that there was a statistically significant interaction of time and group, based on the escape latency of the MWM, between control and sevoflurane-treated mice. B. Platform crossing times were reduced in mice (P34) that were exposed to $3 \%$ sevoflurane for $2 \mathrm{~h}$ daily from $\mathrm{P} 6$ to $\mathrm{P} 8$ (control, $\mathrm{N}=15$; sevoflurane, $\mathrm{N}=15$ ). $\mathrm{C}$. Sevoflurane upregulated the expression of FAS as compared to the control condition in hippocampal tissues. ${ }^{*} P<0.05,{ }^{* *} P<0.01$.

\section{FAS and FASL-knockout attenuated sevoflurane-induced learning and memory impairment in 6-day-old mice}

Next, we investigated whether FAS- and FASL-knockout can mitigate sevofluranerelated learning and memory impairment in mice. We tested the mice by MWM from P30 to P 34. The interaction between time and group, based on the escape latency of the MWM, was statistically significant between the sevoflurane and FAS-knockout groups, as demonstrated by two-way ANOVA with repeated measurement analysis (Figure $2 A)(P=0.022$, two-way ANOVA with repeated measurement). The post hoc Bonferroni test shows that the mice that received sevoflurane anesthesia had longer escape latency than the mice following the FAS-knockout group on P33 and P34. Furthermore, the platform crossing times between sevoflurane $(\mathrm{N}=15)$ (median, 4; interquartile range, 3-5) and FAS-knockout groups ( $N=15)$ (median, 5.2; interquartile range, 4.98) were significantly different (Figure $2 B)(P=0.031$, Mann-Whitney test). The interaction between time and group, based on the escape latency of the MWM, was statistically significant between the sevoflurane and FASL-knockout groups, as demonstrated by two-way ANOVA with repeated measurement analysis (Figure $2 \mathrm{C})(\mathrm{P}=0.034$, two-way ANOVA with repeated measurement). The post hoc Bonferroni test showed that the mice that received sevoflurane anesthesia had longer escape latency than the mice following the FASL-knockout group on P33 and P34. Furthermore, the platform crossing times between sevoflurane $(N=15)$ (median, 3.4; interquartile range, 2.8-5.6) 
and FASL-knockout groups ( $N=15)$ (median, 6; interquartile range, 5-8) were significantly different (Figure 2D) $(P=0.027$, Mann-Whitney test).

A

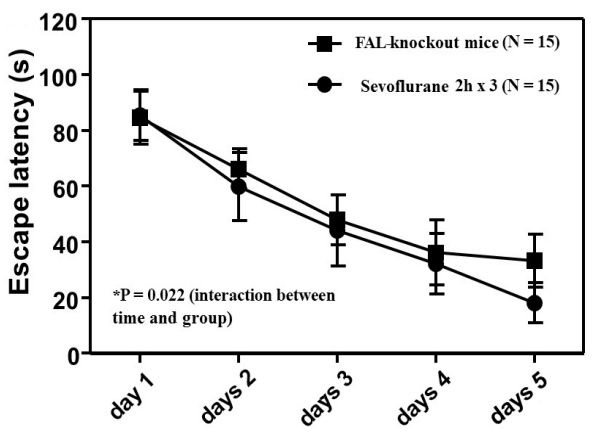

C

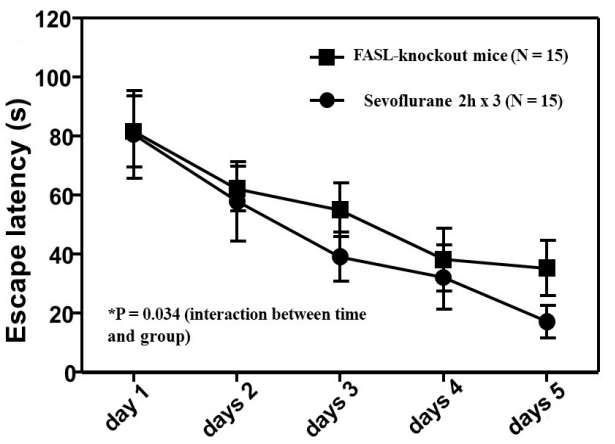

B

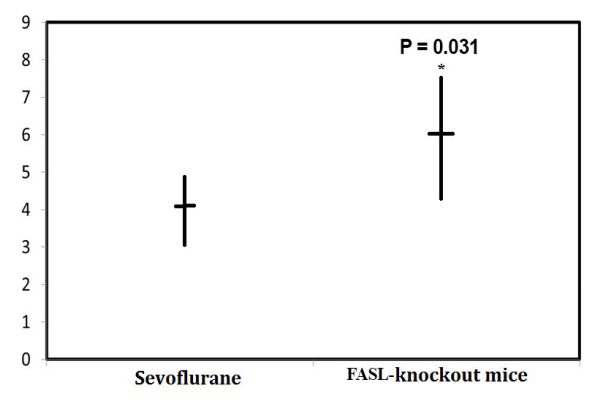

D

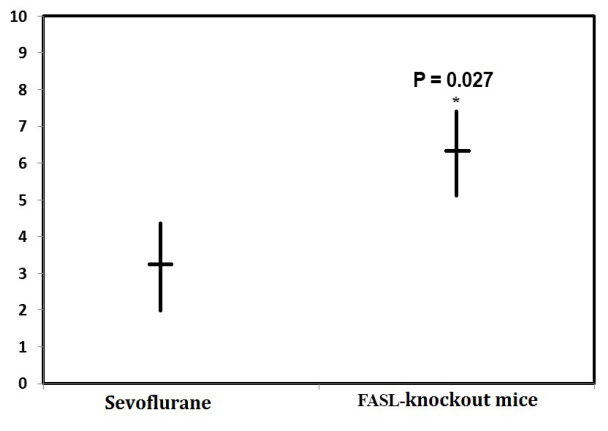

Figure 2. FAS- and FASL-knockout mice attenuated sevoflurane-induced learning and memory impairment in 6-day-old mice. The interaction between time and group, based on the escape latency of the MWM, was statistically significant between the sevoflurane and FAS-knockout groups, as demonstrated by two-way ANOVA with repeated measurement analysis $(\mathbf{A})(P=0.022$, two-way ANOVA with repeated measurement). The post hoc Bonferroni test shows that mice that received sevoflurane anesthesia had longer escape latency than mice following the FAS-knockout group on P33 and P34. Furthermore, the platform crossing times between sevoflurane ( $\mathrm{N}=15)$ (median, 4; interquartile range, 3-5) and FAS-knockout groups ( $\mathrm{N}=15)$ (median, 5.2; interquartile range, 4.9-8) were significantly different $(B)(P=0.031$, Mann-Whitney test). The interaction between time and group, based on the escape latency of the MWM, was statistically significant between the sevoflurane and FASL-knockout groups, as demonstrated by two-way ANOVA with repeated measurement analysis $(\mathbf{C})(P=0.034$, two-way ANOVA with repeated measurement). The post hoc Bonferroni test shows that mice that received sevoflurane anesthesia had longer escape latency than mice following the FASL-knockout group on P33 and P34. Furthermore, the platform crossing times between sevoflurane $(\mathrm{N}=15)$ (median, 3.4; interquartile range, 2.8-5.6) and FASL-knockout groups $(N=15)$ (median, 6; interquartile range, 5-8) were significantly different (D) $\left(P=0.027\right.$, Mann-Whitney test). ${ }^{*} P<$ $0.05,{ }^{* *} \mathrm{P}<0.01 ; \mathrm{MWM}=$ Morris water maze.

\section{FAS-knockout attenuated sevoflurane-induced neuron apoptosis and neurogenesis inhibition in 6-day-old mice}

We have previously shown that FAS-knockout can attenuate sevoflurane-induced learning 
and memory impairment in mice. However, the mechanism by which this occurs is unclear. In this study, we aimed to investigate whether FAS-knockout can mitigate sevoflurane-related neurotoxicity in mice. As expected, sevoflurane induced neuronal apoptosis (Figure $3 \mathrm{~A}$ and $\mathrm{B}$ ) and dramatically decreased the number of BrdU-positive cells in the hippocampus of sevoflurane-treated mice, as compared to control mice (Figure $3 \mathrm{E}$ and F). FAS-knockout attenuated sevoflurane-induced neuronal apoptosis and neurogenesis inhibition (Figure 3C, D, G-J). These results suggest that FAS-knockout attenuates the sevoflurane-mediated induction of neuronal apoptosis and neurogenesis inhibition in mice.
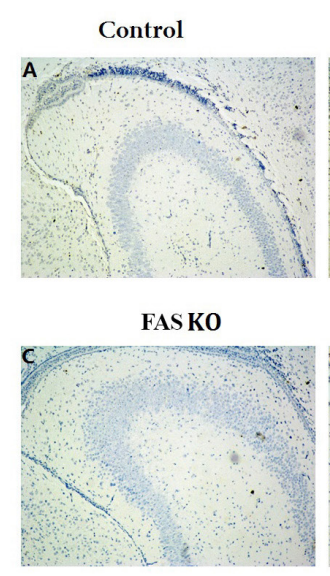

Control

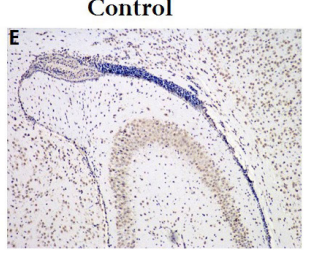

FAS KO

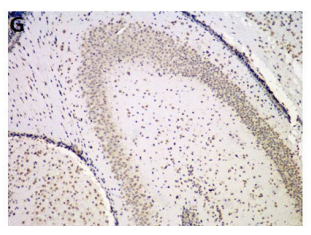

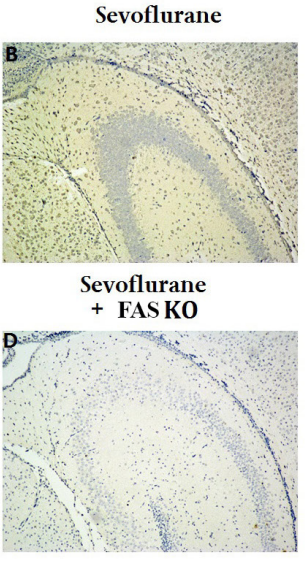

Sevoflurane

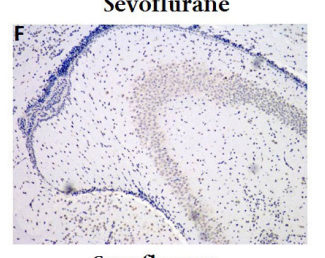

Sevoflurane

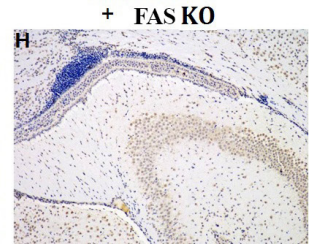

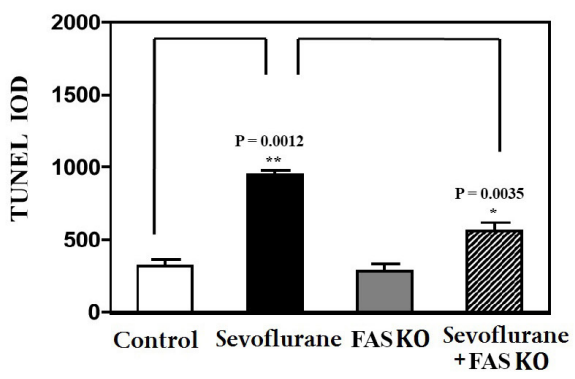

J



Figure 3. FAS-knockout mice attenuated sevoflurane-induced neuron apoptosis and neurogenesis inhibition in 6-dayold mice. A. and B. Anesthesia with 3\% sevoflurane for $2 \mathrm{~h}$ daily for 3 days in P6 mice induced neuronal apoptosis. C. and D. FAS-knockout mice attenuated sevoflurane-induced neuron apoptosis. I. There was a statistically significant difference in the number of TUNEL-positive cells between mice that were exposed to sevoflurane and mice that were treated with both sevoflurane and FAS-knockout mice (two-way ANOVA; sevoflurane: $N=15$, sevoflurane+ FASknockout mice: $N=15$ ). $E$. and $F$. Exposure of $P 6$ mice to sevoflurane for $2 \mathrm{~h}$ daily for 3 days induced neurogenesis inhibition. G. and $\mathbf{H}$. FAS-knockout mice attenuated sevoflurane-induced neurogenesis inhibition. J. There was a statistically significant difference in the number of 5-bromodeoxyuridine (BrdU)-positive cells between mice that were exposed to sevoflurane and mice that were treated with both sevoflurane and FAS-knockout mice (two-way ANOVA; sevoflurane: $\mathrm{N}=15$, sevoflurane + FAS-knockout mice: $\mathrm{N}=15)$. $\mathrm{KO}=$ knockout. 


\section{FASL-knockout attenuates sevoflurane-induced neuron apoptosis and neurogenesis inhibition in 6-day-old mice}

We have shown that FAS-knockout can attenuate sevoflurane-induced learning and memory impairment and sevoflurane-related neurotoxicity in mice. FAS and FASL play critical roles in the immune system, in particular in the killing of pathogen-infected target cells and the death of obsolete, potentially deleterious as well as auto-reactive lymphocytes (Strasser et al., 2009). Therefore, we examined the role of FASL-knockout in sevoflurane-related neurotoxicity. Indeed, we also found that FASL-knockout attenuated sevoflurane-induced neuronal apoptosis and neurogenesis inhibition (Figure 4C, D, G-J). These results suggest that the sevoflurane-mediated induction of neuronal apoptosis and neurogenesis inhibition was through FASL-FAS signaling.

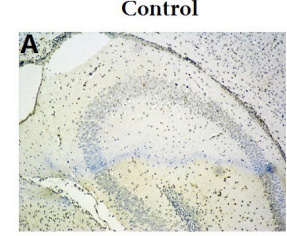

FASL KO

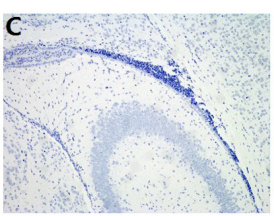

Control

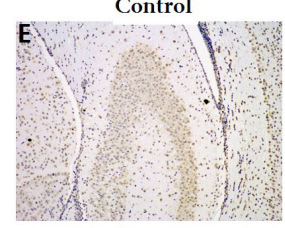

FASL KO

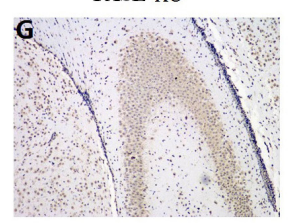

Sevoflurane

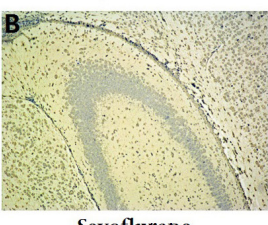

Sevoflurane

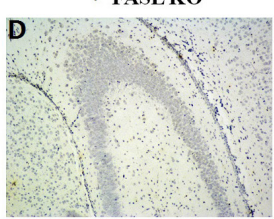

Sevoflurane

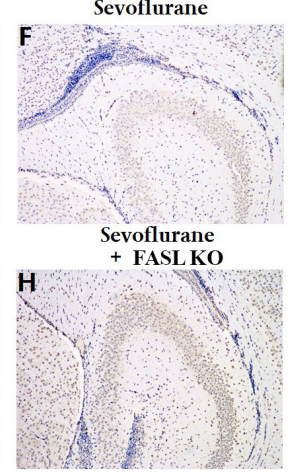

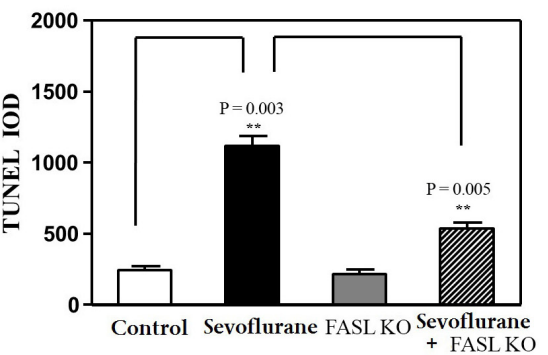

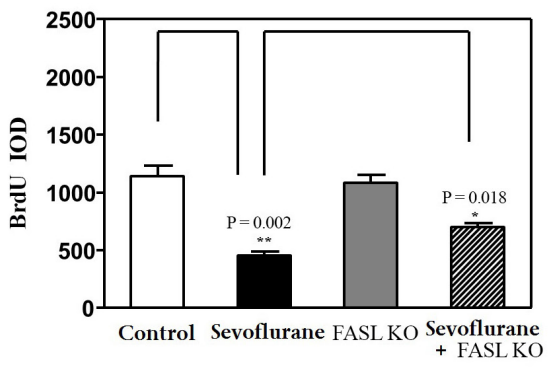

Figure 4. FASL-knockout mice attenuated sevoflurane-induced neuron apoptosis and neurogenesis inhibition in 6-dayold mice. A. and B. Early multiple sevoflurane exposure induced neuronal apoptosis in young mice (P30). C. and D. FASL-knockout mice attenuated sevoflurane-induced neuronal apoptosis. I. The number of TUNEL-positive cells was significantly different between mice that were exposed to sevoflurane and mice that were exposed to both sevoflurane and FASL-knockout mice (two-way ANOVA; sevoflurane: $\mathrm{N}=15$, sevoflurane + FASL-knockout mice: $\mathrm{N}=15$ ). $\mathbf{E}$. and F. Early multiple sevoflurane exposure induced neurogenesis inhibition in young mice (P30). G. and H. FASL-knockout mice attenuated sevoflurane-induced neurogenesis inhibition. J. The number of BrdU-positive cells was significantly different between mice that were exposed to sevoflurane and mice that were exposed to both sevoflurane and FASLknockout mice (two-way ANOVA; sevoflurane: $\mathrm{N}=15$, sevoflurane + FASL-knockout mice: $\mathrm{N}=15$ ). $\mathrm{KO}=$ knockout. 


\section{DISCUSSION}

Sevoflurane, the most widely used anesthetic in clinical practice, has been shown to induce apoptosis, inhibit neurogenesis, and cause learning and memory impairment in young mice (Shen et al., 2013). Children who are exposed to general anesthesia and surgery multiple times at an early age may develop learning and memory impairment. Potential neurotoxicity in the developing brain has engendered considerable concern by the United States Food and Drug Administration (Wang et al., 2009). The hippocampus is known to be important for learning and memory function, and new neurons in the hippocampus of rodents and humans are assumed important for maintaining memory function (Eriksson et al., 1998). Some studies have shown that sevoflurane not only induces neuron apoptosis but also inhibits neurogenesis. However, the underlying mechanism is still unknown (Istaphanous et al., 2011; Lu et al., 2011; Kato et al., 2013).

FAS (also known as APO-1 or CD95) belongs to a subset of the TNF-R family that is involved in death transducing signals and can trigger apoptosis of neurons. Its physiological ligand, FASL (CD95L), is a member of the corresponding TNF cytokine family (Huang et al., 2001). FAS expression can be boosted by cytokines such as interferon and TNF but also by the activation of lymphocytes. FAS-mediated apoptosis is triggered by its cognate ligand, FASL, which is a TNFrelated type II transmembrane molecule and is expressed in a far more restricted way than the receptor (Krammer, 2000). Killer cells (so-called cytotoxic T lymphocytes) remove, for example, virus-infected cells, and those that express FASL can do so by interacting with the FAS receptor on their targets (Strasser et al., 2009).

In the current study, neuronal apoptosis was increased and the number of newborn neurons was decreased in the hippocampus of 6-day-old mice after multiple exposures to sevoflurane. These findings are consistent with other studies and suggest that sevoflurane not only induces apoptosis but also inhibits neurogenesis in the hippocampus of young mice (Shen et al., 2013). Sevoflurane could increase the expression of FAS protein in neurons to activate the signaling pathway inducing apoptosis and inhibiting neurogenesis in the hippocampus. Because of its central role in learning and memory, loss of hippocampal neurons results in learning and memory impairment after sevoflurane treatment. This finding may pave the way for the development of a therapeutic approach to prevent or treat sevoflurane-induced learning and memory impairment.

\section{ACKNOWLEDGMENTS}

Research supported by the Technical Cooperation of the International Division of Zhengzhou (\#20140966).

\section{REFERENCES}

Eriksson PS, Perfilieva E, Björk-Eriksson T, Alborn AM, et al. (1998). Neurogenesis in the adult human hippocampus. Nat. Med. 4: 1313-1317.

Gomez-Sintes R and Lucas JJ (2013). Neuronal apoptosis and motor deficits in mice with genetic inhibition of GSK-3 are Fasdependent. PloS One 8: e70952.

Huang X, Lu Z, Lv Z, Yu T, et al. (2013). The Fas/Fas ligand death receptor pathway contributes to phenylalanine-induced apoptosis in cortical neurons. PloS One 8: e71553.

Istaphanous GK, Howard J, Nan X, Hughes EA, et al. (2011). Comparison of the neuroapoptotic properties of equipotent anesthetic concentrations of desflurane, isoflurane, or sevoflurane in neonatal mice. Anesthesiology 114: 578-587.

Kato R, Tachibana K, Nishimoto N, Hashimoto T, et al. (2013). Neonatal exposure to sevoflurane causes significant suppression 
of hippocampal long-term potentiation in postgrowth rats. Anesth. Analg. 117: 1429-1435.

Krammer PH (2000). CD95's deadly mission in the immune system. Nature 407: 789-795.

Lu Y, Wu X, Dong Y, Xu Z, et al. (2010). Anesthetic sevoflurane causes neurotoxicity differently in neonatal naïve and Alzheimer disease transgenic mice. Anesthesiology 112: 1404-1416.

Medana I, Li Z, Flügel A, Tschopp J, et al. (2001). Fas ligand (CD95L) protects neurons against perforin-mediated T lymphocyte cytotoxicity. J. Immunol. 167: 674-681.

Nikizad H, Yon JH, Carter LB, Jevtovic-Todorovic V (2007). Early exposure to general anesthesia causes significant neuronal deletion in the developing rat brain. Ann. NY. Acad. Sci. 1122: 69-82.

Shen X, Dong Y, Xu Z, Wang H, et al. (2013). Selective anesthesia-induced neuroinflammation in developing mouse brain and cognitive impairment. Anesthesiology 118: 502-515

Strasser A, Jost PJ and Nagata S (2009). The many roles of FAS receptor signaling in the immune system. Immunity 30: 180192.

Wang S, Peretich K, Zhao Y, Liang G, et al. (2009). Anesthesia-induced neurodegeneration in fetal rat brains. Pediatr. Res. 66: 435-440.

Wilder RT, Flick RP, Sprung J, Katusic SK, et al. (2009). Early exposure to anesthesia and learning disabilities in a populationbased birth cohort. Anesthesiology 110: 796-804.

Zhang L, Zhang J, Yang L, Dong Y, et al. (2013a). Isoflurane and sevoflurane increase interleukin-6 levels through the nuclear factor-kappa B pathway in neuroglioma cells. Br. J. Anaesth. 110: i82-i91.

Zhang Y, Dong Y, Zheng H, Shie V, et al. (2013b). Sevoflurane inhibits neurogenesis and the Wnt-catenin signaling pathway in mouse neural progenitor cells. Curr. Mol. Med. 13: 1446-1454.

Zheng H, Dong Y, Xu Z, Crosby G, et al. (2013). Sevoflurane anesthesia in pregnant mice induces neurotoxicity in fetal and offspring mice. Anesthesiology 118: 516-526. 